\title{
Longitudinal Medication Usage in Alzheimer Disease Patients
}

\author{
Carolyn W. Zhu, PhD, ${ }^{* \dagger}$ Elayne E. Livote, MPH, MS, ${ }^{\dagger}$ Kristin Kahle-Wrobleski, PhD, $\ddagger$ \\ Nikolaos Scarmeas, MD, MSc,§\| Marilyn Albert, PhD, 9 Jason Brandt, PhD, 9 \\ Deborah Blacker, MD, ScD,\# Mary Sano, PhD,** and Yaakov Stern, PhD $\S \|$
}

\begin{abstract}
This study examined in detail patterns of cholinesterase inhibitors (ChEIs) and memantine use and explored the relationship between patient characteristics and such use. Patients with probable Alzheimer disease AD $(n=201)$ were recruited from the Predictors Study in 3 academic AD centers and followed from early disease stages for up to 6 years. Random effects logistic regressions were used to examine effects of patient characteristics on ChEIs/ memantine use over time. Independent variables included measures of function, cognition, comorbidities, the presence of extrapyramidal signs, psychotic symptoms, age, sex, and patient's living situation at each interval. Control variables included assessment interval, year of study entry, and site. During a 6-year study period, rate of ChEIs use decreased $(80.6 \%$ to $73.0 \%)$ whereas memantine use increased $(2.0 \%$ to $45.9 \%)$. Random effects logistic regression analyses showed that ChEI use was associated with better function, no psychotic symptoms, and younger age. Memantine use was associated with better function, poorer cognition, living at home, later assessment interval, and later year of study entry. Results suggest that high rate of ChEI use and increasing memantine use over time are consistent with current practice guidelines of initiation of ChEIs in mild-to-moderate AD patients and initiation of memantine in moderate-to-severe patients.
\end{abstract}

Received for publication January 5, 2010; accepted March 20, 2010. From the *This Geriatric Research, Education, and Clinical Center (GRECC) and HSR\&D Center for the Study of Health Care Across Systems and Sites of Care, James J. Peters VA Medical Center, Bronx; †Brookdale Department of Geriatrics, Mount Sinai School of Medicine; \|Gertrude H. Sergievsky Center and the Department of Neurology, Columbia University Medical Center; **Department of Psychology, Mount Sinai School of Medicine; \$Eli Lily \& Company; §Cognitive Neuroscience Division of the Taub Institute for Research in Alzheimer's Disease and the Aging Brain, New York, NY; Department of Psychiatry and Behavioral Sciences, Johns Hopkins University, Baltimore, MD; and \#Department of Psychiatry, Massachusetts General Hospital, Harvard Medical School, Boston, MA.

The Predictors Study is supported by Federal grants AG07370, RR00645, and U01AG010483. Funding for this analysis also was partially provided by Eli Lily \& Company. Drs Zhu and Sano and Ms Livote also are supported by the Department of Veterans Affairs, Veterans Health Administration. The views expressed in this article are those of the investigators and do not necessarily represent the views of the Department of Veterans Affairs. The investigators all certify that they have no conflict of interest to report in this manuscript.

Study concept and design: Zhu, Livote, Kahle-Wrobleski, Scarmeas, Stern. Acquisition of data: Scarmeas, Albert, Brandt, Blacker, Sano, Stern. Analysis and interpretation of data: Zhu, Livote, Kahle-Wrobleski, Scarmeas, Stern. Drafting of manuscript: Zhu. Critical revision of manuscript for important intellectual content: Zhu, Livote, Kahle-Wrobleski, Scarmeas, Brandt, Blacker, Sano, Stern. Statistical analysis: Zhu, Livote.

Reprints: Carolyn W. Zhu, PhD, Geriatric Research, Education, and Clinical Center (GRECC), James J. Peters VA Medical Center, 130 West Kingsbridge Road, Bronx, NY 10468 (e-mail: carolyn. zhu@mssm.edu).

Copyright (C) 2010 by Lippincott Williams \& Wilkins
Key Words: Alzheimer disease, cholinesterase inhibitors, memantine, longitudinal studies

(Alzheimer Dis Assoc Disord 2010;24:354-359)

$A_{b}^{b}$ lzheimer disease (AD) is a degenerative disease of the brain and is the leading cause of dementia in the elderly individuals. Currently there is no cure for AD. Therapeutic strategies aim at treating disease symptoms and delaying the deterioration of cognition and daily functioning. Since their introduction in 1997, cholinesterase inhibitors (ChEIs) have been shown to temporarily stabilize or reduce the rate of deterioration in measures of cognitive function, activities of daily living, and behavior in some patients with mild-tomoderate AD. ${ }^{1}$ Although there is controversy over whether treatment results represent clinically important benefits, ${ }^{2}$ most AD clinical practice guidelines consider ChEIs to be the first-line pharmacotherapy for mild-to-moderate AD. ${ }^{3}$ More recently, a N-methyl-D aspartate receptor antagonist, memantine, was approved for moderate-to-severe dementia. ${ }^{1,4}$ Patterns of using these antidementia treatments recently began to be described in the literature, most focusing on ChEIs. ${ }^{1,5-8}$

In addition to ChEIs and memantine, AD patients are likely to be prescribed other medications, either to address noncognitive symptoms of AD or to manage comorbidities. Polypharmacy is common in elderly persons and has been shown to be associated with adverse events such as increased adverse drug interactions and increased medical care and costs. $^{9-11}$ AD patients are at high risks of polypharmacy because of high rates of comorbidities. ${ }^{12}$ Although guidelines have been developed to identify medications that are inappropriate for use in the elderly, ${ }^{13}$ medication use in AD patients has been described for few drug classes. Among AD patients who were prescribed ChEIs, $17 \%$ to $35 \%$ have been reported to use benzodiazepines, known to worsen cognition, and $24 \%$ to $35 \%$ have been reported to use anticholinergics that directly antagonize their effects. ${ }^{5-7,14}$

Most studies examining use of antidementia and concomitant medications have been based on claims data or data from clinical trials. Although claims data contain detailed information on prescription medications, they often lack clinical information. In contrast, although clinical trials contain detailed clinical and prescription information, patients included in clinical trials are followed for only a short period of time and are often different from those seen in real-world practices and limits the generalizability of study results. ${ }^{15,16}$

This study uses data from the Predictors Study, a large, multicenter cohort of patients with probable $\mathrm{AD}$, followed from early disease stages for up to 6 years 
to examine (1) patterns of ChEIs/memantine use, (2) relationship between patient characteristics and ChEIs/ memantine use, and (3) patterns of concomitant medication use. Our study design allows for substantially longer-term analysis than can generally be conducted in clinical trials. Substantial clinical information included in the Predictors Study allows for more detailed examination of the relationship between medication use and patient characteristics than what would be possible with claims data.

\section{METHODS}

\section{Sample}

The sample was drawn from the Predictors 2 cohort, consisting of patients recruited from Columbia University Medical Center, Johns Hopkins School of Medicine, and Massachusetts General Hospital. The study was approved by each local Institutional Review Board. The inclusion/ exclusion criteria are fully described elsewhere. ${ }^{17-19}$ In brief, the participants met DSM-III-R criteria for primary degenerative dementia of the Alzheimer type and NINDSADRDA criteria for probable AD. Enrollment required a modified Mini-Mental State examination (mMMS) score $\geq 30$, equivalent to approximately $\geq 16$ on the Folstein Mini-Mental State Examination (MMSE). ${ }^{20,21}$ Clinical diagnosis of $\mathrm{AD}$ has been confirmed in $93 \%$ of those with postmortem evaluation, ${ }^{19}$ suggesting high degrees of certainty in AD diagnosis.

Study recruitment began in 1997 and was staggered: $3 \%(\mathrm{n}=6)$ entered the study in $1997,8.5 \%(\mathrm{n}=17)$ in 1998 , $8.0 \%(\mathrm{n}=16)$ in $1999,26.9 \%(\mathrm{n}=54)$ in $2000,24.4 \%$ $(\mathrm{n}=49)$ in $2001,13.4 \%(\mathrm{n}=27)$ in $2002,10.0 \%(\mathrm{n}=20)$ in 2003 , and $6.0 \%(\mathrm{n}=12)$ in 2004 or later. After the baseline interview, patients were followed annually. Those who did not respond at a particular visit could respond at a subsequent visit. For data used in this analysis, 17 patients had one assessment $(8.5 \%), 41$ had $2(20.4 \%), 32$ had 3 $(15.9 \%), 36$ had $4(17.9 \%), 33$ had $5(16.4 \%)$, and 42 had 6 or more assessments $(20.9 \%)$. Median follow-up for the cohort was 4 years. Differences in the number of assessments during follow-up reflect both continuous accrual of patients and patient deaths $(7 \%)$. Missed visits during follow-up were rare: $15.6 \%$ missed 1 visit, $2.5 \%$ missed 2 , and $1 \%$ missed 3 visits. The analysis sample consisted of 785 observations from 201 patients.

\section{Measures}

\section{Clinical Characteristics}

Data on clinical characteristics of the patients were recorded at each visit. Disease progression was characterized by transition from milder stages of dementia to more severe stages, measured by MMSE. ${ }^{20}$ Higher MMSE scores indicate better cognition. Blessed Dementia Rating Scale (BDRS) Parts I (Instrumental Activities of Daily Living) and II (Basic Activities of Daily Living) were used to assess patients' functional capacity. ${ }^{22}$ Higher BDRS scores indicate worse functioning. Columbia University Scale for Psychopathology in AD (CUSPAD), a semi-structured interview administered by physicians or trained research technicians, was used to measure psychotic symptoms. ${ }^{23} \mathrm{We}$ constructed a dichotomous indicator for the presence of psychotic symptoms if the patient had any delusions, hallucinations, or illusions. A modified Unified Parkinson's Disease Rating Scale (UPDRS) was used to measure the presence of extrapyramidal signs (EPS). ${ }^{24,25}$ We constructed a dichotomous indicator for the presence of EPS if any of the following 11 items was rated 2 or higher (with 0 being normal and 4 maximum impairment): speech, facial expression, tremor at rest, neck rigidity, right arm rigidity, left arm rigidity, right leg rigidity, left leg rigidity, posture, gait, or bradykinesia. Patients' medical histories were used to construct a modified Charlson comorbidity index. ${ }^{19,26}$

\section{Sociodemographic Characteristics}

At baseline, demographic characteristics (eg, age, ethnicity, sex, education) were recorded. Information on living arrangements was collected at each visit, dichotomized as living at home or in a long-term care facility (ie, retirement home, assisted living facility, or nursing home).

\section{Use of ChEIs and Memantine}

All patient/caregiver report of prescription and over the counter (OTC) medications use were recorded at each visit on a medication acquisition form. As the ChEIs have been shown to have similar efficacy despite slightly different pharmacological properties, we constructed a dichotomous variable indicating any ChEIs use, and a separate dichotomous variable indicating memantine use. To examine concurrent ChEIs/memantine use, we also constructed indicators for using (1) ChEIs only, (2) memantine only, (3) both ChEIs and memantine, or (4) neither ChEIs nor memantine. Although tacrine is also approved by the FDA for mild-to-moderate $\mathrm{AD}$, it is rarely used owing to hepatoxicity. No patient in the sample reported using tacrine.

\section{Year of Study Entry}

The ChEIs and memantine were approved by the FDA for treatment of AD at different times. Specifically, among ChEIs, donepezil was approved for treatment of mildto-moderate AD on November 25, 1996 (the label was expanded to include severe AD in 2007), rivastigmine was approved for treatment of mild-to-moderate AD on April 21, 2000, and galantamine was approved for treatment of mild-to-moderate AD on February 28, 2001. More recently, memantine was approved by the FDA for treatment of moderate-to-severe AD on October 16, 2003. Year of study entry was included to control for availability of medications on the market.

\section{Concomitant Medications Use}

In addition to ChEIs and memantine, patients and informants reported using a total of 429 unique prescription medications and 176 OTC medications. A neurologist who specializes in dementia (N.S.) categorized all prescription medications into the following 18 categories: anticoagulants, antiplatelets, nonsteroidal antiinflamatory drugs (NSAIDs), antihistamine, prostate-anticholinergic, for dyslipidemia, antidiabetics, antihypertensives, antiepileptics, antipsychotics, antiemetic neuroleptics, stimulants, for Parkinson disease, antidepressants, benzodiazepines, narcotics, hormones, and other prescription medications. All OTC drugs were categorized as either vitamins or other. For each drug category, we constructed a dichotomous variable indicating whether a patient reported using any medications in that drug category at each visit. A complete list of medications by category is available upon request from the investigators. 


\section{Analysis}

Random effects logistic regression models were used to estimate the effects of patient characteristics on ChEIs/ memantine use over time. ${ }^{27}$ This framework allows an exploration of the combination of fixed effects that are common to all individuals in the population or common to groups of individuals and random effects that indicate individual-level variations. The fixed effects parameters are interpreted as average effects of each explanatory variable on the dependent variables. The random effects are interpreted as deviations from the mean for each individual, and therefore model the magnitude of unobserved heterogeneity.

In this regression model, time was measured in years following baseline (year 0 ). The coefficient on year estimates average linear trend in ChEIs/memantine use over time. We also included a year-squared term in the model to estimate the rate of change over time. For example, an odds ratio of $>1$ on the coefficient on time indicates increasing likelihood of use over time. An odds ratio $>1$ on the yearsquared term indicates that the rate of change estimated by the linear term is accelerating over time.

All clinical variables were entered as time-variant covariates except for Charlson comorbidity index because there was little change in the measure over time. Demographic variables except living arrangement were entered as time-invariant covariates. As the sample was overwhelmingly white $(93 \%)$, we did not include race as an explanatory variable.

We controlled for the effects of availability of medications on the market by including year of study entry. We also controlled for possible differences in use patterns by region by including indicators for study sites. All analyses were done using Stata 9.0.28

\section{RESULTS}

\section{Baseline Characteristics}

At baseline, patients' average age was $76(\mathrm{SD}=8.1)$, $61 \%$ were female, $93 \%$ were white, and $84 \%$ lived at home (Table 1, first column). Patients were highly educated, with an average of 14 years of schooling $(\mathrm{SD}=3.1)$. Average MMSE was $22.0(\mathrm{SD}=3.5)$ and average BDRS was 4.8 $(\mathrm{SD}=2.5)$. Psychotic symptoms $(34 \%)$ and extrapyramidal signs $(16 \%)$ were common. On average, patients had 1.5 comorbidities $(\mathrm{SD}=1.1)$, with $15 \%$ with none, $43 \%$ with 1 , $26 \%$ with 2 , and $16 \%$ with 3 or more comorbidities.

\section{Unadjusted Results on ChEls and Memantine Use Over Time}

Figure 1 shows rates of ChEIs/memantine use over time. At baseline, $80.6 \%$ of the patients used ChEIs; $2.0 \%$ memantine. By year 6 , the proportion of patients who used ChEIs decreased to $73.0 \%(P=0.003)$; and the proportion of patients who used memantine increased to $45.9 \%$ $(P<0.001)$. The proportion of patients who used specific ChEIs remained relatively stable (all $P>0.05$ ), with an average of $61.7 \%$ reported using donepezil, $4.8 \%$ rivastigmine, and $13.6 \%$ galantamine. Examination of concurrent ChEIs/memantine use showed that, over time, whereas the proportion of patients who used neither ChEIs nor memantine remained relatively stable at approximately $20 \%$, the proportion of patients who used ChEIs-only decreased from $78.6 \%$ to $32.4 \%$, those who used both ChEIs and memantine increased from $2.0 \%$ to $40.5 \%$, and those who used memantine-only increased from $0 \%$ to $5.4 \%$.

\section{Multivariate Results on Relationship Between Patient Characteristics and ChEls and Memantine Use}

Table 1 also presents results of random effects logistic regression models of the effects of patient characteristics on ChEIs/memantine use. The second column of Table 1 shows that patients with worse functioning were less likely to use ChEIs $(\mathrm{OR}=0.83, P<0.05)$, as were patients with psychotic symptoms $(\mathrm{OR}=0.47, P<0.05)$ or those who were older $(\mathrm{OR}=0.86, P<0.001)$. After controlling for year of study entry, the probability of using ChEIs increased over time $(\mathrm{OR}=1.29, P<0.05)$. The third column of Table 1 shows that patients with worse functioning were less likely to

TABLE 1. Baseline Characteristics and Random Effects Model of ChEls and Memantine Use Over Time

\begin{tabular}{|c|c|c|c|}
\hline & Baseline Characteristics Mean (s.d) & ChEIs OR (s.e.) & Memantine OR (s.e.) \\
\hline Functional capacity, BDRS & $4.8(2.5)$ & $0.8337(0.080)^{*}$ & $0.7134(0.101)^{* *}$ \\
\hline Folstein MMSE & $22.0(3.5)$ & $1.0618(0.045)$ & $0.8062(0.046)^{* * *}$ \\
\hline Extrapyramidal signs (\%) & 15.5 & $1.0463(0.506)$ & $1.8716(1.208)$ \\
\hline Psychotic symptoms (\%) & 33.8 & $0.4662(0.212)^{*}$ & $0.6582(0.408)$ \\
\hline Charlson comorbidity Index & $1.5(1.1)$ & $0.8575(0.193)$ & $0.7211(0.230)$ \\
\hline Female $(\%)$ & 61.2 & $0.4398(0.253)$ & $0.3628(0.252)$ \\
\hline Baseline age & $76.3(8.1)$ & $0.8647(0.036)^{* * *}$ & $1.0006(0.047)$ \\
\hline Live at home $(\%)$ & 84.0 & $2.0245(1.145)$ & $6.817(5.773)^{* *}$ \\
\hline \multicolumn{4}{|l|}{ Site $($ reference group $=$ Columbia $)$} \\
\hline Johns Hopkins ( $\%)$ & 25.4 & $2.1374(1.568)$ & $0.712(0.678)$ \\
\hline Massachusetts General (\%) & 28.4 & $0.4192(0.272)$ & $1.8689(1.470)$ \\
\hline Year of entry into study & $2001(1.9)$ & $1.0529(0.163)$ & $6.8229(1.973) * * *$ \\
\hline Interval & - & $1.2866(0.202)^{*}$ & $5.2686(1.828)^{* * *}$ \\
\hline Interval squared & - & $0.9835(0.014)$ & $0.9487(0.023)^{* *}$ \\
\hline $\mathrm{AIC}$ & - & 417.426 & 271.696 \\
\hline $\mathrm{BIC}$ & - & 482.58 & 336.85 \\
\hline log likelihood & - & -193.71 & -120.85 \\
\hline
\end{tabular}




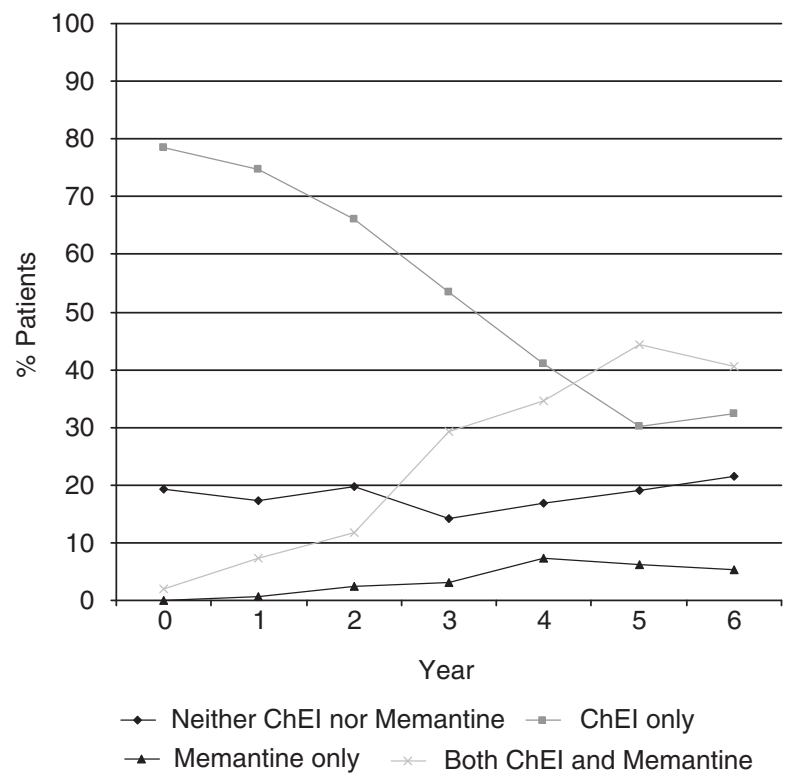

FIGURE 1. Use of ChEl and memantine over time.

use memantine $(\mathrm{OR}=0.71, P<0.05)$, as were those with better cognition (OR $=0.81, P<0.001)$. Living at home was significantly associated with higher likelihood of using memantine $(\mathrm{OR}=6.82, P<0.01)$. Later entry year was significantly associated with higher likelihood of using memantine $(\mathrm{OR}=6.82, P<0.001)$. After controlling for entry year, patients were more likely to use memantine over time $(\mathrm{OR}=5.27, P<0.001)$, although the rate of increase slowed over time $(\mathrm{OR}=0.95, P<0.01)$.

In a secondary analysis, we controlled for the effect of concurrent use of memantine in the ChEIs equation. Results showed that concurrent use of ChEIs did not have a significant effect on memantine use. The effect of concurrent use of ChEIs in the equation estimating memantine use also was statistically insignificant.

\section{Unadjusted Results on Patterns of Other Medications Use Over Time}

On average, patients reported 4.3 prescription medications at each interval. These medications belonged to 2.1 drug categories at baseline, increasing to 2.6 in year 6 $(P=0.004)$. We computed the percentage of patients who were on medications by drug category at each visit (Fig. 2). For all assessment intervals, the most frequently used medications included antihypertensives (overall average use rate $=53.0 \%$ ), antiplatelets (overall average use rate $=$ $35.2 \%$ ), antidepressants (overall average use rate $=37.8 \%$ ), and medications for dyslipidemia (overall average use rate $=31.3 \%$ ). Tests for trend over time in these 4 drug categories using random effects logistic regressions show increases in the use rates of antihypertensives and antidepressants (both $P<0.001$ ) but not in medications for dyslipidemia and antiplatelets.

Use rates for 2 additional categories of medications changed substantially over time. Specifically, hormone use steadily decreased from $19.4 \%$ at baseline to $5.4 \%$ in year 6 , and antipsychotics use increased steadily from $3.5 \%$ at baseline to $27.0 \%$ in year 6 (both $P$-values for trend over time $<0.001)$.

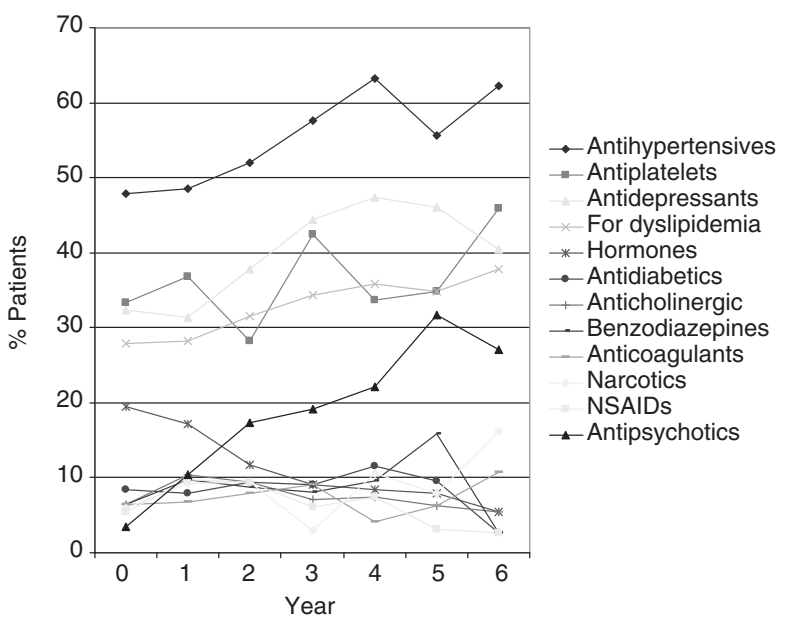

FIGURE 2. Use of other medications by category over time.

Other prescription medications were used less frequently, with an average overall rate ranging from $7.0 \%$ for anticoagulants and NSAIDs to $8.8 \%$ for antidiabetics. With few exceptions, use rate of 5 categories of drugs (ie, antihistamines, antiepileptics, Parkinson disease medications, stimulants, and antiemetic neuroleptics) was lower than $5 \%$ at all assessment intervals.

Use of other prescription and OTC medications were common. At each assessment interval, vitamins were the most frequently used, although utilization rate decreased from $79.1 \%$ at baseline to $64.9 \%$ in year $6(P=0.002)$, use of other prescription medications increased from $46.3 \%$ to $64.9 \%(P<0.001)$, and other OTC medications fluctuated between $39.7 \%$ and $48.8 \%(P>0.05)$.

\section{DISCUSSION}

In this study, we prospectively followed a large cohort of patients from early stages of AD and examined patterns of ChEIs/memantine use over 6 years. This study extends the literature in several fronts: (1) by examining patterns of ChEIs/memantine use simultaneously, (2) by using multivariate analysis to explore the longitudinal relationship between patient characteristics and ChEIs/memantine use more rigorously, and (3) by describing patterns of concomitant medication use more comprehensively.

Similar to recent studies that examined ChEIs use among dementia patients, ${ }^{6-8,29,30}$ we found high rates of ChEIs use, with donepezil being the most prevalent agent. Over time, use patterns changed substantially. At baseline, almost $80 \%$ of the patients used ChEIs only and none used memantine; by year 6 , almost half of the patients used both ChEIs and memantine. These use patterns suggest adherence to current practice guidelines of initiating a ChEIs in milder stages of $\mathrm{AD}$, followed by initiation of memantine in moderate to severe stages of the disease. At each assessment interval, however, approximately $20 \%$ of the patients were not using either ChEIs or memantine. The reason for not using any antidementia therapies should be examined in future studies.

The relationship between ChEIs use and several demographic characteristics have been explored recently. ${ }^{6,30}$ In this study, ChEI use was associated with younger age but memantine use was not significantly associated with age. These inconsistent results suggest the need for replication 
with other samples. Similar to previous findings, this study did not find any gender differences in ChEI use.

Effects of clinical characteristics on ChEIs/memantine use have not been examined earlier. Results from our multivariate analyses show that, consistent with current practice guidelines, patients were more likely to use ChEIs and memantine as disease progresses after controlling for availability of the medications. Over and above the chronological development of the disease, use of ChEIs also was associated with better function and not having psychotic symptoms. Our results also show that different clinical characteristics are associated with ChEIs and memantine use. In particular, memantine use was associated with better function, poorer cognition, and patient living at home.

The relationships of both ChEIs/memantine use with better function suggest that patients on these medications receive a functional benefit that results in continued use. Alternatively, physicians may perceive patients with higher functioning as more suitable candidates for ChEIs and memantine use. The association between poorer cognition and memantine use suggests that physicians appropriately use cognitive decline as a benchmark for increasing disease severity and initiate memantine in accordance with practice guidelines.

There may be several reasons for the significant relationship between living at home and higher likelihood of using memantine. On the healthcare demand side, it is likely that compared with institutionalized patients, patients who live at home have caregivers and family members who act as advocates for treatment. On the supply side, professional caregivers for patients living in nursing homes may be less concerned with patients' impaired cognitive status and more concerned with the treatment of their other symptoms and conditions.

Findings from the analyses suggest high rates of concomitant medication usage in this sample of AD patients. Notably, the reported use of antihypertensive agents, antidepressants, and antipsychotics increased significantly over time. The increase in antidepressant and antipsychotics use suggests that the treatment of dementia symptoms is not limited to ChEIs and memantine. Further analyses looking at behavioral symptoms of AD and their association with rates of antidepressant and antipsychotics use may help characterize the spectrum of pharmacologic treatment for dementia. Among the patients who used antipsychotics, the vast majority $(95 \%)$ used atypical antipsychotics. On account of the small number of patients who used typical antipsychotics, we combined these drugs into one class and did not test the sensitivity of our results separating typical and atypical antipsychotics.

There are several limitations to our study. First, patients were selected from tertiary care university hospitals and specialized AD centers and represent a nonrandom sample of those affected by the disease in the population. Patients in our sample also were predominantly white and highly educated. Caution is needed in generalizing the results of this study to patients of other ethnicities, patients at lower education and income levels, and to community AD patients. Second, because of the different approval dates of medications, we controlled for year of study entry and interval in the analyses. However, we were unable to take into account the uptake curve of new pharmaceutical agents onto the market or availability on specific formularies. As such, the effect of availability may be underestimated for patients entering the study near the time of
FDA approval. In contrast, because patients were selected from specialized AD centers, careful clinical diagnosis of $\mathrm{AD}$ and evaluations permit more reliable and complete data and more accurate coefficient estimates. Uptake of new pharmaceutical agents by the physicians in these specialized AD centers may be faster than in the general community. The magnitude of the possible underestimation of the effects on drug availability may be lessened. As the patients were recruited at early disease stages and followed for long periods of time, analyses are not compressed in time and the cohort describes the full range of progression. Longer-term effects are therefore more easily interpreted and strengthen the confidence in our findings.

\section{REFERENCES}

1. Birks J. Cholinesterase inhibitors for Alzheimer's disease. Cochrane Database Syst Rev. 2009:CD005593.

2. National Institute for Clinical Excellence. Technology appraisal guidance 111: Donepezil, rivastigmine, galantamine (review) and memantine for the treatment of Alzheimer's Disease (amended). 2007 [cited 2009 April 10, 2009]; Available from: http://www.nice.org/uk.

3. Lyketsos CG, Colenda CC, Beck C, et al. Position statement of the American Association for Geriatric Psychiatry regarding principles of care for patients with dementia resulting from Alzheimer disease. Am J Geriatr Psychiatry. 2006;14:561-572.

4. McShane R, Areosa S, Minakaran N. Memantine for dementia. Cochrane Database Syst Rev. 2006;2.(CD003154).

5. Carnahan RM, Lund BC, Perry PJ, et al. The concurrent use of anticholinergics and cholinesterase inhibitors: rare event or common practice? J Am Geriatr Soc. 2004;52:2082-2087.

6. Dybicz SB, Keohane DJ, Erwin WG, et al. Patterns of cholinesterase-inhibitor use in the nursing home setting: a retrospective analysis. Am J Geriatr Pharmacother. 2006;4: 154-160.

7. Herrmann N, Gill SS, Bell CM, et al. A population-based study of cholinesterase inhibitor use for dementia. $\mathrm{J} \mathrm{Am}$ Geriatr Soc. 2007;55:1517-1523.

8. Mucha L, Shaohung S, Cuffel B, et al. Comparison of cholinesterase inhibitor utilization patterns and associated health care costs in Alzheimer's disease. J Manag Care Pharm. 2008; 14:451-461.

9. Chiang L, Hirsch SH, Reuben DB. Predictors of medication prescription in nursing homes. J Am Med Dir Assoc. 2000; 1:97-102.

10. Perri M III, Menon AM, Deshpande AD, et al. Adverse outcomes associated with inappropriate drug use in nursing homes. Ann Pharmacother. 2005;39:405-411.

11. Haider SI, Johnell $\mathrm{K}$, Thorslund $\mathrm{M}$, et al. Trends in polypharmacy and potential drug-drug interactions across educational groups in elderly patients in Sweden for the period 1992-2002. Int J Clin Pharmacol Ther. 2007;45:643-653.

12. Zhao Y, Kuo T-C, Weir S, et al. Healthcare costs and utilization for Medicare beneficiaries with Alzheimer's. BMS Health Services Research. 2008;8.

13. Fick DM, Cooper JW, Wade WE, et al. Updating the Beers criteria for potentially inappropriate medication use in older adults: results of a US consensus panel of experts. Arch Intern Med. 2003;163:2716-2724.

14. Fick D, Kolanowski A, Waller J. High prevalence of central nervous system medications in community-dwelling older adults with dementia over a three-year period. Aging Ment Health. 2007;11:588-595.

15. Lee PY, Alexander KP, Hammill BG, et al. Representation of Elderly Persons and Women in Published Randomized Trials of Acute Coronary Syndromes. JAMA. 2001;286:708-713.

16. Tinklenberg JR, Kraemer HC, Yaffe K, et al. Donepezil treatment and Alzheimer disease: can the results of randomized clinical trials be applied to Alzheimer disease patients in clinical practice? Am J Geriatr Psychiatry. 2007;15:953-960. 
17. Stern Y, Folstein M, Albert M, et al. Multicenter study of predictors of disease course in Alzheimer disease (the "Predictors Study"). I. Study design, cohort description, and intersite comparisons. Alzheimer Dis Assoc Disord. 1993; 7:3-21.

18. Richards M, Folstein M, Albert M, et al. Multicenter study of predictors of disease course in Alzheimer disease (the "Predictors Study"). II. Neurological, psychiatric, and demographic influences on baseline measures of disease severity. Alzheimer Dis Assoc Disord. 1993;7:22-32.

19. Scarmeas N, Hadjigeorgiou GM, Papadimitriou A, et al. Motor signs during the course of Alzheimer disease. Neurology. 2004;63:975-982.

20. Folstein MF, Folstein SE, McHugh PR. "Mini-mental state". A practical method for grading the cognitive state of patients for the clinician. J Psychiatr Res. 1975;12:189-198.

21. Stern Y, Sano M, Paulson J, et al. Modified mini-mental state examination: validity and reliability. Neurology. 1987; 37(suppl 1): 179 .

22. Blessed G, Tomlinson BE, Roth M. The association between quantitative measures of dementia and of senile change in the cerebral grey matter of elderly subjects. $\mathrm{Br} J$ Psychiatry. 1968;114:797-811.

23. Devanand DP, Miller L, Richards M, et al. The Columbia University Scale for Psychopathology in Alzheimer's disease. Arch Neurol. 1992;49:371-376.
24. Stern MB, Hurting HI. The Clinical Characteristics of Parkinson's Disease and Parkinsonian Syndromes: Diagnosis and Assessment, in The Comprehensive Management of Parkinson's Disease. New York: PMA Corp; 1978; $3-50$.

25. Richards M, Marder K, Bell K, et al. Interrater reliability of extrapyramidal signs in a group assessed for dementia. Arch Neurol. 1991;48:1147-1149.

26. Charlson ME, Pompei P, Ales KL, et al. A new method of classifying prognostic comorbidity in longitudinal studies: development and validation. J Chronic Dis. 1987;40: 373-383.

27. Rabe-Hesketh S, Skrondal A. Multilevel and Longitudinal Modeling Using Stata. College Station, TX: Stata Press; 2005.

28. Statacorp. Stata Statistical Software: Release 9. StataCorp LP: College Station, Texas; 2005.

29. Doody RS, Geldmacher DS, Gordon B, et al. Open-label, multicenter, phase 3 extension study of the safety and efficacy of donepezil in patients with Alzheimer disease. Arch Neurol. 2001;58:427-433.

30. Vidal JS, Lacombe JM, Dartigues JF, et al. Memantine therapy for Alzheimer disease in real-world practice: an observational study in a large representative sample of French patients. Alzheimer Dis Assoc Disord. 2008;22: 125-130. 